\title{
Tangence
}

\section{Quelques notes sur la critique-femme}

\section{Louise Dupré}

Numéro 51, mai 1996

Paradigmes critiques

URI : https://id.erudit.org/iderudit/025909ar

DOI : https://doi.org/10.7202/025909ar

Aller au sommaire du numéro

Éditeur(s)

Tangence

ISSN

0226-9554 (imprimé)

1710-0305 (numérique)

Découvrir la revue

Citer cet article

Dupré, L. (1996). Quelques notes sur la critique-femme. Tangence, (51), 144-156. https://doi.org/10.7202/025909ar d'utilisation que vous pouvez consulter en ligne.

https://apropos.erudit.org/fr/usagers/politique-dutilisation/ 


\section{Quelques notes sur la critique-femme Louise Dupré}

J'avais d'abord pensé refuser cette invitation. Ces dernières années, j'ai en effet publié une suite d'articles sur la critiquefemme, de sorte que j'ai l'impression d'avoir transmis l'essentiel de ma réflexion ${ }^{1}$. Si j'ai accepté, c'est que j'ai fait un pari : arriver, dans ce texte, à ne pas trop me répéter, avancer dans les trous que j'ai laissés dans mes bilans précédents, me donner la permission d'être parfois irrévérencieuse, parler à l'intérieur même de la contradiction. Ou plutôt laisser parler la contradiction. Car il me semble que ce n'est que du choc des idées qu'un certain inédit peut actuellement advenir. Aussi avancerai-je dans ma réflexion sous forme de notes que le lecteur, la lectrice pourra lire à la suite l'une de l'autre ou disposer côte à côte, sur une table de travail, pour créer d'autres configurations, d'autres corrélations. Pour proposer une autre vision.

\section{Note 1}

La critique-femme a obtenu, au cours de la dernière décennie, ses lettres de noblesse. On peut affirmer qu'aujourd'hui, ses objets et méthodes sont reconnus par l'ensemble de la communauté universitaire. Elle a réussi à créer une demande. Elle est parvenue à s'immiscer dans le marché de la recherche. Du moins en théorie, car est-ce bien vrai dans la pratique? Si elle est acceptée, c'est encore en tant que critique marginale, qui opère sur des

1 Cet article est en effet le quatrième volet d'une série de réflexions sur la critique-femme. On pourra lire à ce sujet le premier volet, "La critique au féminin : réalité et utopie ", dans Claudine Potvin et Janice Williamson (dir.), Women's Writing and the Literary Institution/Lecriture au féminin et l'institution littéraire, Edmonton, University of Alberta, Research Institute for Comparative Literature, 1992, p. 69-77; le deuxième volet, "La critique-femme, esquisse d'un parcours", dans Annette Hayward et Agnès Whitfield (dir.), Critique et littérature québécoise, Montréal, Triptyque, 1992, p. 397-406; et le troisième volet, "La critique au féminin", dans Claude Duchet et Stéphane Vachon (dir), La recherche littéraire, objets et métbodes, Paris et Montréal, Presses de l'Université de Vincennes et XYZ éditeur, 1993, p. 379-386. 
corpus féminins. On reconnaîtra rarement que son point de vue a opéré une véritable coupure épistémologique, que ses apports nous obligent à repenser tout le champ critique. On continuera à faire tranquillement ses travaux sans se soucier d'intégrer à sa démarche des ouvrages portant sur la question de la sexuation de l'écriture.

Il en est de même dans les départements. Si aucun cours n'est proposé, il se crée rapidement un vide théorique auquel personne ne pensera suppléer. Le département continuera de tourner, sans malaise, sans qu'on s'inquiète de la formation des étudiants et étudiantes. On compte sur les professeures inscrites dans ce champ de recherche pour mettre sur pied des cours appropriés, sinon rien n'est fait. Voilà qui m'amène à corriger mon affirmation: la demande est là, bien sûr, tant et aussi longtemps que l'offre la précède, bien visible. Quand celle-ci vient à manquer, le besoin s'estompe rapidement.

Il marrive de penser que ce champ d'étude pourrait disparaître de nos universités, si on ne s'assure pas que les étudiants des deuxième et troisième cycles voient l'importance d'une telle approche. Car qui fera les engagements dans les universités de demain? Qui sera en poste? Qui déterminera les priorités? L'expérience nous montre que c'est durant les études qu'on se familiarise avec un domaine de recherche, rarement après. Un jeune professeur qui n'aura jamais reçu de cours en études féministes ne verra pas la nécessité de défendre cet axe. C'est dire à quel point il est important de consolider ce champ dans les universités. De travailler à la formation d'une "relève".

Les études féministes ne sont pas un caprice de femmes qui, depuis les années 1970, se cherchent une cause. Ce n'est pas un effet de mode, mais un domaine de recherche en plein développement.

\section{Note 2}

La grandeur et la misère de la critique-femme tiennent à l'investissement émotif chez celles qui la touchent de près. En effet, les femmes - car la plupart du temps, ce sont des femmes qui la pratiquent - s'engagent dans une démarche qui n'est pas étrangère à leur quête d'identité. On a affaire à un axe d'études où le sujet de l'énonciation n'est pas coupé de l'objet de l'énoncé. On 
146

pourrait alléguer que tout discours critique est teinté de la subjectivité qui le produit. Mais on reconnaîtra que d'autres types de critique offrent une distance plus confortable, permettant une certaine objectivité dans l'énonciation: une sémiotique de type greimassien, par exemple, en serait l'exemple idéal.

La critique-femme n'est pas neutre, et elle le sait. Cette implication, cette passion même quelquefois, chez l'énonciatrice, n'est pas sans produire d'effets, non seulement sur le style, le rythme, le ton des écrits, mais aussi sur le contenu. Dans un premier temps, une première période de la critique, certains textes se sont voulus engagés, voire polémiques. Le mot femme a donné lieu à des recherches productives. Mais il a donné lieu aussi à des généralisations, voire aussi à certaines naïvetés, à des affirmations qui tenaient parfois davantage de l'utopie que de la réalité. Non pas qu'il faille rejeter cette critique, mais la replacer dans son contexte historique, politique. La lire au deuxième degré.

Depuis les années 1980, la critique-femme a trouvé une plus grande objectivité, suite au développement des recherches basées sur des approches théoriques. On a vu de plus en plus apparaitre des articles proposant des lectures inédites. Cette critique dégage des pistes fructueuses, non seulement pour les études féministes, mais aussi pour les autres domaines de recherche. C'est cette voie qui a donné aux études féministes ses assises durables, sa crédibilité. Car si, dans un premier temps, une vision "militante" a pu produire un dynamisme certain, elle ne pouvait, à long terme, qu'enliser la critique dans l'“idéologique”. Et cela n'aurait pas servi la pensée.

\section{Note 3}

La critique-femme vient, rappelons-le, de la jonction entre deux domaines de recherche: le développement de la théorie féministe dans le champ des sciences humaines - l'histoire, la sociologie, la psychanalyse, etc. - et le passage du structuralisme au poststructuralisme, qui a replacé le texte dans sa chaîne énonciative. Rendue possible, sur le plan littéraire, par la diffusion des théories de l'énonciation, la critique-femme a contribué à réhabiliter la notion d'auteur, à la repenser, à la redéfinir. Elle a remis en question la mort de l'auteur, l'effacement du sujet, elle a réitéré le lien étroit existant entre le sujet et son énoncé, à partir de l'affirmation suivante: le sujet n'est pas neutre. "Parler n'est 
jamais neutre ${ }^{2}$, écrivait Luce Irigaray. En effet, le sujet est soumis aux contingences de l'Histoire, la grande, comme de sa petite histoire. Et la différence sexuelle reste un des pivots - le plus important sans doute - autour desquels se forme la subjectivité. La femme entretient un rapport différent au symbolique, au langage. Comme le rappelle Antoinette Fouque, la "différence sexuelle conditionne une différence de destin, en ce qu'elle n'est pas uniquement une différence anatomique, parce qu'elle se trouve accueillie dans ce qu'on appelle l'ordre symbolique" 3 .

Il y aurait donc des traces d'une subjectivité féminine dans la langue. Il n'est pas question pourtant de parler d'une languefemme, d'une écriture-femme comme étant "naturelles" aux femmes, mais de rapport, de médiation, ce qui ne facilite pas l'analyse, du point de vue méthodologique. D'autre part, si la différence sexuelle est le principal pôle de différenciation chez l'individu - du moins dans l'Histoire, et c'est encore le cas dans nos sociétés actuelles -, il n'occulte pas tous les autres: les particularités ethniques, sociales, religieuses, générationnelles, familiales sont autant de traits différentiels autour desquels se constitue la personnalité de l'individu. Et, dans le domaine des sciences humaines, ces traits sont moins facilement isolables qu'un gène, pour le biologiste, dans un laboratoire.

Aujourd'hui, on admet qu'il n'est pas possible de réunir, sous le dénominateur commun d'écriture au féminin, les textes des femmes du Tiers-Monde et ceux des Occidentales, ceux des femmes noires américaines et ceux des femmes blanches. On admet que la textualité est un tissu de différences complexes. Aussi ne parle-t-on plus de L'Écriture au féminin comme s'il s'agissait d'une entité homogène et, par là, descriptible, préhensible.

Mais on reconnaît également - et c'est peut-être ce qu'il y a de plus important pour nous, littéraires - qu'une écriture véritable n'est jamais parfaitement réductible à la différence sexuelle qui la produit, ni à son environnement, ni à sa génération. Une écriture n'est pas un reflet de la réalité sociale: c'est une activité par laquelle le sujet échappe à son identité fixe pour établir des

2 Luce Irigaray, Parler n'est jamais neutre, Paris, Minuit, 1985.

3 Antoinette Fouque, ll y a deux sexes, Paris, Gallimard, coll. "Le débat ", 1995, p. 175 . 
148

rapports avec une altérité. Une écriture est toujours singulière. C'est le lieu d'un passage constant entre le même et l'autre. Le lieu d'un dépassement de ses origines.

\section{Note 4}

Car la critique-femme travaille justement dans le champ littéraire, là où le lien entre énonciation et énoncé est brouillé, là où l'énonciateur ne se donne pas comme maître de son énoncé, où il se déguise, se travestit, accepte d'être "un autre". Espace de jeu, de leurre, le texte littéraire est celui d'une remise en question de la subjectivité, le lieu où le sujet se construit en se déconstruisant.

Qui parle en effet dans le poème? Dans le texte dramatique? Dans le roman? Est-ce le même énonciateur que dans la conversation? Ou dans l'essai? Cette question a passionné et passionne encore les théoriciens. Barthes, on s'en souvient, s'est intéressé à cette question à propos de Sarrazine, de Balzac ${ }^{4}$. Un spécialiste de la génétique littéraire, Louis Hay, s'est attardé à cette "instance écrivante, tendue entre le vécu et la feuille blanche " 5 que rencontre nécessairement le critique dans son travail. De son côté, Robbe-Grillet, s'interrogeant sur l'autobiographie, affirmait qu'il n'avait jamais parlé d'autre chose que de lui-même ${ }^{6}$. On peut le constater: la question du statut de l'énonciateur dans le texte littéraire a pris ces dernières années une importance grandissante, avec le retour à la notion d'auteur: elle traverse tout le champ des études littéraires. Mais elle a une incidence capitale pour une critique qui suppose la sexuation du discours. On ne peut en faire l'économie. Elle reste au centre des préoccupations des chercheures qui travaillent dans une perspective féministe.

Elle doit cependant être sans cesse mise en relation avec le statut du texte littéraire d'une part et, d'autre part, avec une poétique des genres. Ceci est primordial quand on se propose d'étudier la subjectivité dans les œuvres, plus particulièrement dans les œuvres contemporaines, qui cherchent à brouiller les pistes des conventions génériques, associant la fiction et la réflexion de type

4 Roland Barthes, $S / Z$, Paris, Seuil, coll. "Tel", 1970, p. 178.

5 Louis Hay, "Le texte n'existe pas", Poétique, n' 62, avril 1985, p. 156.

6 Alain Robbe-Grillet, "Je n'ai jamais parlé d'autre chose que de moi", dans Michel Contat (dir.), L'auteur et le manuscrit, Paris, Presses Universitaires de France, coll. "Perspectives critiques", 1991, p. 37-50. 
essayistique, le romanesque et le poétique, parfois le polémique. Dans tous ces livres qu'on a nommés "textes", "écriture fragmentaire", "nouvelle écriture", "fiction-théorie" ou "théorie-fiction", à défaut de pouvoir les classer dans des cases bien définies. Dans ces textes de femmes qui ont voulu repenser la notion de genre.

En effet, par cette mixité, ce mélange des genres, les écrivaines ont trouvé, ces dernières années, une façon de remettre en question les genres traditionnellement masculins et la possibilité, la liberté de repenser le champ du littéraire, de l'ouvrir à la généricité - l'autre, oui, celle du "gender" -, en même temps que de faire avancer la pensée sur le féminin. Car il y a habituellement un lien entre "gender and genre", selon l'expression de nos collègues anglophones. C'est par là que doit débuter une analyse littéraire. Par le type de discours où s'inscrit l'énonciation.

\section{Note 5}

En effet, qui parle? Le narrateur, faut-il le répéter, n'étant pas la figure de l'auteur, ne peut-on pas supposer qu'il est possible de dépasser les configurations de son "gender", de s'imaginer autre et, par conséquent, d'imaginer une narration du point de vue de l'autre sexe? " $M^{\text {me }}$ Bovary, c'est moi ", disait Gustave Flaubert. Sans lire cette affirmation au premier degré, ne peut-on pas penser qu'il y ait glissement vers une altérité qui ne soit pas appropriation de l'autre? Inclusion de l'autre par le même? Cela nous amène à poser une autre question: le féminin est-il le propre de la femme? On se rappellera qu'Hélène Cixous, dans son célèbre article "Le rire de la Méduse", plaçait Jean Genet parmi les auteurs féminins ${ }^{7}$.

Qu'est-ce que le féminin, au fait? Des théoriciennes, des théoriciens ont répondu, chacun à sa façon: Beauvoir, Lacan, Kristeva, Derrida, Irigaray, de Lauretis, Butler, Jardine, Silverman. Plus près de nous, Suzanne Lamy, Patricia Smart, pour ne nommer que quelques noms. Les points de vue se sont multipliés, rencontrés, affrontés, passant d'un point de vue psychanalytique à un point de vue plus sociologique, de la théorie pure à des

7 Hélène Cixous, "Le rire de la Méduse ", L'arc, $n^{\circ} 61,1975$, p. 47. Je la cite: "Hâtons-nous: le continent n'est pas d'un noir impénétrable. J'y suis souvent allée. J'y ai un jour rencontré avec joie Jean Genet [... ] Il y a des hommes (si peu) qui n'ont pas peur de la féminité. 
approches plus pragmatiques. Mais nous n'avons pas encore de réponse claire, définitive. Et l'on sait toutes les discussions qu'ont entraînées les réflexions autour de l'essentialisme...

La question est rendue plus complexe encore avec la montée des lesbian studies, des queer studies, par les possibilités qu'offre aujourd'hui la science de se construire autre. Nous sommes dans une civilisation en pleine mutation, dans une remise en question des valeurs et une incroyable créativité technologique qui déplace le réel vers le virtuel, ce qui entraîne une révision des concepts qui fondent notre réflexion. On ne peut faire comme si cela n'existait pas. Voilà sans doute pourquoi on rencontre actuellement, chez les femmes, une résistance à la théorisation globalisante. Il y a moins de risques si on travaille "sur le terrain", dans le concret des textes de femmes. Position moins ambitieuse, mais certes plus confortable. Small is beautiful.

Ce qu'on peut tout de même affirmer, c'est cela : il y a du féminin dans les textes de femmes. Surtout à notre époque où celles-ci sont moins obligées de se travestir pour publier, où elles ne sont plus obligées d'adopter un autre point de vue sur le langage, sur l'écriture. Mais bien avant aussi. On le découvre en réouvrant l'histoire litteraire, en y faisant des fouilles. Si tant de femmes ont été occultées, c'est que leurs œuvres ne cadraient pas dans les attentes institutionnelles de leur époque, de leur "milieu" littéraire. Il y a un débordement, un trop-plein dans beaucoup d'œuvres de femmes. Une délinquance, un non-respect de la mesure. Comme si la barre entre signifiant et signifié était plus poreuse chez la femme que chez l'homme. Il s'agit du rapport au symbolique, encore une fois. Du rapport à la castration, ce terme qui a souvent choqué. J'oserais ceci : considérées comme étrangères dans la phratrie, les femmes peuvent entrer dans la langue en inventant, elles dérangent l'ordre signifiant plus facilement que les, hommes. Même si elles veulent imiter le Père, elles n'y arrivent jamais parfaitement: il reste toujours une maille dans le tricot, qui leur rappelle leurs origines. Les écrivains masculins transgressent eux aussi, mais autrement. Et cet acte leur demande une énorme dépense d'énergie, qui se retourne parfois contre eux. Faut-il nommer Artaud? Gauvreau? Aquin? On ne déroge pas impunément à la Loi du Père tout-puissant.

Mais je fais ici de la théorie. Et pourtant, mon expérience des textes me dit que je n'ai pas tout à fait tort. On pourrait bien sûr me donner des exemples qui démontreraient le contraire. Il y a 
toujours une femme qui arrive à entrer dans les paramètres masculins, à adopter ces modes de fonctionnement. Voilà pourquoi la critique est plus confortable. Travaillant sur un corpus réduit, on a moins devant les yeux l'exception qui, confirmant la règle, donne pourtant l'impression qu'elle l'infirme.

\section{Note 6}

J'ai écrit expérience. Pour souligner que l'énonciatrice, la personne qui parle dans la théorie, déteint sur son discours. Même si elle essaie de masquer les marques de son énonciation, elle se trahit en interprétant le texte: n'est-ce pas la condition sine qua non d'une bonne lecture? On sait pourtant que la critique sérieuse, celle qui refuse l'impressionnisme, tente pourtant de donner à son discours toutes les marques d'une objectivité minimale. Cet état de fait, souhaitable d'un certain point de vue - je l'ai dit —, est d'autant plus vrai depuis que le modèle scientifique s'est imposé dans le champ des sciences humaines, depuis que le mot critique est de plus en plus souvent remplacé par le mot recbercbe, façon de montrer à nos collègues les scientifiques, les vrais, que nous ne sommes pas tout à fait farfelus, que notre discours vaut, qu'il mérite d'être entendu... et subventionné.

J'oserai pourtant un autre mot, qui ne fait pas sérieux: le mot intuition. Je ne suis pas la première à l'employer. Je citerai une chercheure au-dessus de tout soupçon, Catherine KerbratOrecchioni: "Pour effectuer le repérage des unités qu'il nous semble légitime de considérer comme subjectives, nous nous fierons avant tout, il faut l'avouer sans ambages, à notre propre intuition." 8 Voilà un mot presque tabou, actuellement, dans le domaine littéraire. Et pourtant, on admettra que, tout aussi rigoureux qu'ils soient, certains chercheurs ont de l'intuition et savent renouveler le discours sur un sujet, alors que d'autres reprennent les discours existants. Certains chercheurs sont ce qu'on pourrait appeler des penseurs alors que d'autres, non. Très vite nous savons repérer les uns et les autres chez nos étudiants.

La critique-femme n'échappe pas à la règle. Je réitérerai, à son propos, une affirmation qu'avait faite en 1978 Joseph

8 Catherine Kerbrat-Orecchioni, L'énonciation. De la subjectivité dans le langage, Paris, Armand Colin, 1980, p. 71. 
Bonenfant: "L'analyse linguistique de la poésie ne donne pas de l'intelligence à celui qui n'en a pas, mais elle n'en ôte pas non plus à celui qui en a." ${ }^{9}$ C'est bien toujours de cela qu'il s'agit. Aussi affirmerai-je que, dans le domaine de la critique-femme comme dans tous les autres domaines, il y a aussi des textes qui reproduisent les discours reçus sans les mettre à l'épreuve de la pensée, comme il y a des textes qui cherchent à faire avancer la réflexion sur le féminin. Les premiers sont utiles pour diffuser une œuvre, ne nous le cachons pas, mais ce sont les derniers qui sont le plus intéressants: non seulement ils ouvrent des perspectives neuves pour la lecture d'une œuvre, mais aussi pour la théorie. Et la critique-femme a besoin de ces textes qui dégagent des voies nouvelles, ces textes qui ne se laissent pas arrêter par un discours codifié, un "prêt-à-porter " du discours sur le féminin.

\section{Note 7}

Comment nommer cette critique qui se penche sur des corpus produits par des femmes à partir de théories du féminin? Jusqu'à maintenant, $j$ 'ai employé le terme critique-femme, mais les chercheures françaises la nomment critique féminine, alors que, en Amérique du Nord, on parle plutôt de critique féministe. La nuance n'est pas sans conséquences, on en conviendra. Elle révèle des différences dans l'attitude, l'engagement politique, voire l'approche méthodologique à la base des études: une approche davantage fondée sur la psychanalyse en France et sur la sociologie aux États-Unis. Quant à nous, chercheures québécoises, si nous avons été influencées d'abord par les théories françaises, nous nous sommes tournées ensuite vers les théories américaines, de sorte que le "gender" est maintenant au centre de notre discours. D'où parfois un certain inconfort dans notre énonciation - de quel lieu parlons-nous? -, mais aussi la possibilité de penser une critique métisse, qui tienne de l'un et de l'autre courants. Une critique originale, créative.

Je ne le cacherai pas: tout en reconnaissant l'apport inestimable des recherches françaises et américaines, je ne me sens parfaitement à l'aise ni avec la vision d'un féminin derridien ou

9 Joseph Bonenfant, "L'analyse linguistique en poésie", Revrue de l'Université laurentienne, vol. X, $\mathrm{n}^{\circ} 2$, février 1978, p. 35. 
kristevien, signifiant flottant qui se donnerait en dehors du symbolique: le féminin passe aussi par la signifiance. Une signifiance ayant une configuration différente peut-être, mais une signifiance, tout de même. Je ne me sens pas plus à l'aise avec le féminin considéré comme pur produit de sa réalité historique et sociale: le sujet porte bien sûr les marques du contexte d'où il est issu, mais il est aussi un être de résistance et d'invention. Cela me semble particulièrement important pour nous qui étudions la chose littéraire, ce lieu travaillé par l'idéologie, mais qui la travaille aussi, la déconcerte.

Aussi est-ce la raison qui me fait adopter le terme critique au féminin pour parler d'une vision qui tienne de plusieurs approches à la fois, qui essaie de jouer là où les différents champs théoriques peuvent se rejoindre, mais aussi où ils se manquent, laissent des failles, des ouvertures pour la pensée. Une critique qui accepte de lire à chaque fois le texte avec des yeux volontairement naïfs, remettant chaque fois en question les grilles, les découpages qui fondent les a priori théoriques. Cette critique au féminin appartient aux études féministes. Mais tout en étant sa cellule subversive, parfois jusqu'à la rébellion, elle en est son pôle interne d'avancée. De renouvellement. Elle essaie de lire autrement les marques du féminin. Elle essaie de lire autrement. Elle essaie de lire.

\section{Note 8}

Car la critique au féminin permet de faire avancer la théorie sur le féminin. En travaillant sur le singulier d'une œuvre, sur sa singularité, elle opère le lien entre le particulier et le général, elle crée des brèches dans la totalité, elle nous force à voir la théorie comme une domaine toujours miné, jamais à l'abri d'un corps étranger qui la ronge et nous oblige à repenser le tout à partir de la partie. Elle fonctionnerait comme ces virus informatiques, ces maladies qui envahissent un système logique et le dérèglent.

Car c'est bien de logique qu'il s'agit. La critique au féminin ne peut se construire que dans une logique de l'aporie, en pensant ensemble les contraires qui ne s'annulent pas, qui cohabitent dans un même système. Ainsi cette hypothèse: une écriture de femme est et n'est pas une écriture féminine. Une écriture d'homme est et n'est pas une écriture d'homme. N'est pas une écriture féminine. N'est pas qu'une écriture masculine. Dans le 
154

laboratoire textuel, les choses sont plus complexes qu'elles ne paraissent de prime abord.

Cela veut-il dire que toute généralisation devrait être abolie? Même si nous le désirions, nous ne le pourrions pas. Aussi ajouterai-je que la théorie féministe telle qu'elle a été pensée jusqu'à maintenant nous est essentielle, qu'elle construit des catégories qui nous permettent de découper le champ conceptuel pour mieux appréhender la réalité. Ce sur quoi je veux insister, c'est sur ses limites. Comme sur les limites d'une critique qui ne s'en dégagerait pas suffisamment.

Un exemple. Je suis de ceux et celles qui tiennent le roman Si par une nuit d'biver un voyageur d'Italo Calvino comme un des très beaux romans des dernières décennies. J'avais beaucoup ri à la lecture du portrait qu'il faisait, avec l'humour qu'on lui connaît, de Ludmilla et Lotaria, deux jeunes filles opposées: la première, la lectrice, était la figure même de la souplesse, de la nuance, alors que l'autre, l'universitaire, la féministe, appliquait au texte littéraire des grilles qui le faisaient parler dans le sens qu'elle souhaitait. Or, dans un article intitulé "Calvino and the Amazons " 10 , Teresa de Lauretis, une théoricienne dont j'estime les travaux, a démontré que l'auteur avait repris, dans son roman, les stéréotypes sur le féminin: Ludmilla était la bonne fille et Lotaria, la mauvaise, la "méchante", le modèle à ne pas imiter. J'ai terminé l'article, je l'avoue, en éprouvant un certaine malaise.

En construisant ses personnages féminins, Italo Calvino "reproduisait" les idées reçues sur la féminité. Nul doute que, d'un point de vue idéologique, Teresa de Lauretis a raison: cette Lotaria n'est pas présentée comme un personnage positif, nuancé. En créant le personnage de Lotaria, Italo Calvino règle bien sûr ses comptes avec un féminisme de la ligne juste. Mais n'aurait-il pas fallu voir que, se plaçant du côté de Ludmilla, Calvino adhère à ce féminin dont parlent des théoriciennes comme Cixous, Irigaray ou Montrelay? Il me semble aussi que la chercheure a raté une autre dimension de ce roman. Ludmilla et Lotaria sont en effet les figures de deux types de critique du texte littéraire. Calvino interroge précisément, chez Lotaria, un certain type de

10 Teresa de Lauretis, "Calvino and the Amazons", Tecbnologies of Gender: Essays on Tbeory, Film and Fiction, Bloomington, Indiana University Press, 1987 , p. $70-83$. 
lecture comme mode de savoir rigide, qui découpe la réalité textuelle en parties parfaitement étanches les unes aux autres, la met dans des cases repérables, descriptibles, comptabilisables. Quant à Ludmilla, elle représente cette lecture ouverte que plusieurs Québécoises et Canadiennes ont tenté de mettre en place dans leurs articles, ces dernières décennies. Bref, j'ai le sentiment que Si par une nuit d'biver un voyageur n'a pas été lu d'un point de vue littéraire. Le roman n'a pas été lu comme un roman.

\section{Note 9}

Tout en appartenant à ce qu'il convient d'appeler maintenant la recherche, au sens large, la critique au féminin pose finalement la question de la spécificité de la critique littéraire comme discours. Elle nécessite, de celui qui la pratique, une posture d'écrivain plutôt que d'écrivant. Ainsi André Brochu la voit-il comme une "écriture", une "poésie d'idées" qui tente de "rendre compte de la spécificité du texte littéraire", de "la vérité de l'écriture "11. Je retiendrai le mot poésie comme langage axé sur le processus primaire, fonctionnant à partir d'associations libres, admettant les contraires sans les annuler, laissant passer le pulsionnel, travaillant la figure, accueillant la part d'indicible dans tout langage.

Cette "poésie d'idées" s'appuierait donc sur un mode de fonctionnement susceptible de déroger à la logique binaire, de faire des associations qui ne sont pas évidentes, d'admettre à la fois le vrai et le faux inhérent à tout énoncé, d'adopter le point de vue du texte littéraire, moins en le mimant pourtant qu'en en faisant ressortir les multiples facettes, la complexité. On comprendra pourtant qu'il ne s'agit pas de sacrifier la rigueur: il n'est pas possible de travailler en dehors de la logique discursive - cette "poésie d'idées" n'est pas de la poésie. Il s'agit plutôt de privilégier une autre rigueur, inventive et souple, une autre logique, qui apprivoise la part d'inconscient à l'œuvre dans le texte littéraire et qui travaille avec cette part, non contre elle ou en dehors d'elle. Il s'agit de montrer, dans l'articulation même du texte critique, le travail de l'aporie, de la fragmentation, du silence, de l'indicible. Une telle critique implique que l'on reconnaisse, dans un texte, dans la signature même d'un auteur, ce qui échappe au nom

11 François Dumont, "De plain-pied dans l'écriture. Entretien avec André Brochu ", Voix et images, vol. XX, no 3 (60), printemps 1995, p. 520. 
156

propre, ce qui tient de l'ombre ${ }^{12}$, selon le terme de Michèle Montrelay.

Dans Éperons, Jacques Derrida écrivait que toute écriture est féminine ${ }^{13}$. Si on accepte cette affirmation, on peut avancer qu'il y a du féminin dans la critique. Que celle-ci, comme écriture, serait la part féminine de la recherche. Cela voudrait-il dire que toute critique est critique au féminin? Dans sa méthode, oui, peut-être. Mais la critique au féminin travaille à partir d'un savoir qui, mettant en relation le conscient et l'inconscient, se donne comme savoir du féminin. Du féminin comme lieu de résistance face à la prédominance d'un découpage discursif de type logocentrique. Du féminin comme conscience d'un inconscient critique, de la critique comme écriture. En ce sens, la critique au féminin n'est pas seulement l'affaire des femmes qui travaillent dans le champ féministe. Elle concerne tous les universitaires qui, sans enlever à la recherche la part qui lui revient, veulent assurer l'avenir de la critique comme avenir même d'une lecture spécifiquement littéraire.

12 Michèle Montrelay, L'ombre et le nom, Paris, Minuit, 1977.

13 Jacques Derrida, Éperons. Les styles de Nietzsche. Paris, Flammarion, 1978. 